\title{
Immunotherapy of tumors with human telomerase reverse transcriptase immortalized human umbilical vein endothelial cells
}

\author{
XIYAN MU ${ }^{1,2}$, YAXIONG SANG $^{2}$, CHUNJU FANG $^{2}$, BIN SHAO $^{2}$, LU YANG $^{1}$, KUI YAO $^{1}$, \\ XITONG ZHAO $^{1}$, JINHAI GOU ${ }^{1}$, YUQUAN WEI ${ }^{2}$, TAO YI $^{1}$, YANG WU ${ }^{2}$ and XIA ZHAO ${ }^{1}$ \\ ${ }^{1}$ Department of Gynecology and Obstetrics, West China Second Hospital, Sichuan University, Chengdu, Sichuan 610041; \\ ${ }^{2}$ State Key Laboratory of Biotherapy and Cancer Center, West China Hospital, Sichuan University, \\ and Collaborative Innovation Center for Biotherapy, Chengdu, Sichuan 610041, P.R. China
}

Received June 24, 2015; Accepted August 19, 2015

DOI: $10.3892 /$ ijo.2015.3175

\begin{abstract}
Human umbilical endothelial cells (HUVECs) have been proven to be effective in tumor anti-angiogenesis but the mechanism remained to be further demonstrated. The restricted ability of HUVECs to proliferate in vitro also limits their application on a large scale. In the present study, we immortalized HUVECs with hTERT genes by lentiviral infection and explored the antitumor immunity of hTERTexpressing HUVECs (HUVEC-TERTs). Results showed that HUVEC-TERTs maintained high telomere activity and expressed CD31, VEGFR-II and integrin $\alpha 5$. Passage-30 HUVEC-TERTs were able to form vascular tubes in vitro without showing signs of senescence. In vivo HUVEC-TERTs elicited antitumor immunity in mouse LL2 and CT26 models protectively and therapeutically. Both humoral and cellular immunity participated in the tumor anti-angiogenesis as HUVEC-neutralizing sera antibodies and HUVEC-specific CTL were detected. The subsets of activated spleen T lymphocytes included both $\mathrm{CD} 4^{+} \mathrm{T}$ cells and $\mathrm{CD} 8^{+} \mathrm{T}$ cells. Moreover, MDSCs and Tregs were decreased while $\mathrm{T}$ lymphocytes were aggregated in the tumor microenvironment. Collectively, the present study is the first to confirm the antitumor immunity of hTERT-immortalized HUVECs. Both anti-angiogenesis and tumor microenvironmental regulation participated in the antitumor activity. Transducing hTERT genes might be a new strategy to allow HUVECs to be applied on a large scale in cancer immunotherapy.
\end{abstract}

Correspondence to: Dr Xia Zhao, Department of Gynecology and Obstetrics, West China Second Hospital, Sichuan University, No. 20, Section 3, South People's Road, Chengdu, Sichuan 610041, P.R. China

E-mail: drzhaoxia@163.com; xia-zhao@126.com

Dr Yang Wu, State Key Laboratory of Biotherapy and Cancer Center, West China Hospital, Sichuan University, and Collaborative Innovation Center for Biotherapy, No. 17, Section 3, South People's Road, Chengdu, Sichuan 610041, P.R. China

E-mail: wuyang@scu.edu.cn

Key words: immortalization, hTERT, human umbilical endothelial cells, immunotherapy, microenvironment

\section{Introduction}

Cancer vaccines aim to stimulate a host immune response that leads to tumor repression. Traditional cancer vaccines target tumor-associated antigens. However, this is very challenging since tumor cells of various tissue origins are not only different but also genetically instable (1). Thus, researchers have been focusing on anti-angiogenic vaccines because tumor vascular endothelium is genetically stable and shows similar properties in different cancer types (2). Moreover, anti-angiogenic therapy is useful in combination therapies for increasing tumor sensitivity to chemotherapy and radiotherapy (3). Anti-angiogenic therapies can be divided into two types. One is the application of monoclonal antibodies or synthetic molecules against angiogenesis-associated antigens (4). The other is the whole endothelial vaccine. Recent studies have proven that vaccination with HUVECs could prevent tumors by attacking on tumor vasculature with both cellular and humoral immunity $(5,6)$. One pilot study treated patients who had recurrence of their brain tumors or metastatic colorectal cancer with glutaraldehyde-fixed HUVECs and found specific cellular immune responses against HUVECs. Partial or complete tumor responses for at least 9 months were shown in three patients with malignant brain tumors (7).

However, primary HUVECs, the most widely used cells in anti-angiogenic immunity, have a very limited ability to proliferate in vitro. They enter a growth arrest known as replicative senescence after a certain number of cell divisions (8). Senescent cells experience both morphological changes and functional losses (9). This makes it difficult to apply HUVECs on a large scale. Attempts to extend the life span of HUVECs include spontaneous transformation, ectopic expression of viral oncogenes, the provision of supportive matrix components and ectopic expression of the human telomerase reverse transcriptase (hTERT) gene (10-12). Several studies have proven that hTERT-immortalized endothelial cells exhibited functional and morphogenetic characteristics of parental cells while displaying a survival advantage beyond the hurdle of replicative senescence $(13,14)$. However, whether hTERTimmortalized HUVECs could preserve antitumor immunity has not yet been confirmed. In the present study, we immortalized primary HUVECs by virally introducing hTERT genes, 
which could renew replicative capacity by means of telomere maintenance via the de novo synthesis of telomeric DNA (15). We also explored whether the antitumor immunity could be maintained through vaccination with hTERT-immortalized HUVECs.

\section{Materials and methods}

Cell culture. Primary HUVECs were isolated from human umbilical cord veins with $0.1 \%$ collagenase treatment and were cultured in endothelial cell growth media (EBM-2) including $0.1 \%$ hEGF, $0.04 \%$ hydrocortisone, $0.1 \%$ CA- $1000,2 \%$ FBS, $0.4 \%$ hFGF-B, $0.1 \%$ VEGF, $0.1 \%$ R3-IGF-1, $0.1 \%$ heparin and $0.1 \%$ ascorbic acid (Lonza, Basel, Switzerland). The purity of extracted HUVECs was identified by flow cytometric analysis to compare the expression of specific endothelial marker CD31 with that of HUV-EC-Cs, an established endothelial cell line (American Type Culture Collection, Manassas, VA, USA). HUVECs were passaged twice a week at a split ratio of 1:3-5 by TrypLE ${ }^{\mathrm{TM}}$ Select (Gibco, Waltham, MA, USA) digestion.

Recombinant vector creation and lentiviral infection. The cDNA sequence of human telomerase was amplified by PCR with pGRN145 as the template (kindly provided by Dr Wei Wang of our laboratory). The sense primer was 5'-agaaga attcgccaccatgccgcgcgctccccgctgccgagcc-3' with an EcoRI linker at the 5' end; the antisense primer was 5'-aga agcggccgctcagtccaggatg gtcttgaagtc-3' with a NotI linker at the $5^{\prime}$ end. Amplified cDNA was then inserted into pCDH lentivector plasmid at EcoRI/NotI site (System Biosciences, Mountain View, CA, USA). HEK293 cells were transfected by the plasmids of psPAX $\mathrm{X}_{2}, \mathrm{pMD} 2 . \mathrm{G}$ and pCDH-hTERT using Trans $\mathrm{IT}^{\circledR}-2020$ transfection reagents (Mirus Bio LLC, Madison, WI, USA). Forty-eight hours later, viral stocks were harvested from cell supernatants and stored at $-80^{\circ} \mathrm{C}$ in aliquots (Fig. 1A and B).

Lentiviral infection of passage-2 HUVECs was carried out in the presence of $4 \mu \mathrm{g} / \mathrm{ml}$ polybrene (Sigma-Aldrich, St. Louis, MO, USA). Stable hTERT-expressing HUVECs were selected with $0.5 \mu \mathrm{g} / \mathrm{ml}$ puromycin for the first 7 days and afterwards with $0.02 \mu \mathrm{g} / \mathrm{ml}$ puromycin.

Reverse transcription-PCR. Transcriptional expression of telomerase was tested using reverse transcription-PCR. Briefly, total RNA of HUVEC-TERTs and primary HUVECs was extracted with RNeasy ${ }^{\circledR}$ Mini kit (Qiagen Benelux B.V., Venlo, The Netherlands) and reverse transcripted to cDNA with PrimeScript $^{\mathrm{TM}}$ RT-PCR kit (Takara, Dalian, China) following the protocols. PCR was performed with the sense primer of hTERT as 5'-cacctcacccacgcgaaaa-3' and the antisense primer as $5^{\prime}$-ccaaagagtttgcgacgcatgtt-3' (16). The products of RT-PCR were visualized by Southern blot analysis in $1 \%$ soft agarose.

Telomeric repeat amplification protocol assay. Telomerase activity was quantified by Telo TAGGG telomerase PCR ELISA (Roche Diagnostics, Basel, Switzerland). According to the manufacturer's recommendation, $2 \times 10^{5}$ cells for each sample were prepared for cell extracts, $3 \mu \mathrm{l}$ of which were transferred to PCR process for the amplification of telomerase-specific 6 nucleotide increments (TTAGGG). The PCR products were
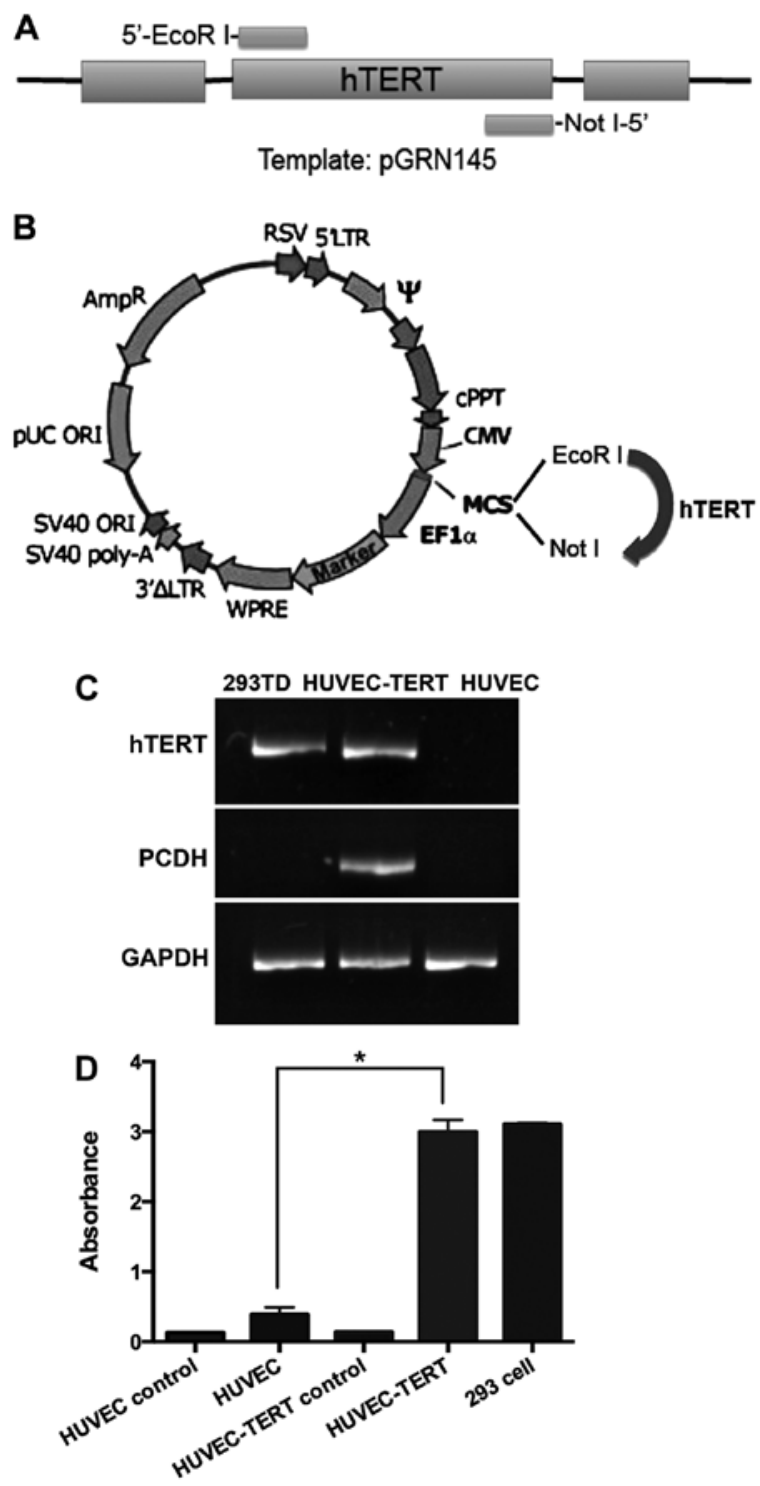

Figure 1. Construction of recombinant lentivirus and detection of telomerase expression. (A) The cDNA sequence of human telomerase was amplified by PCR with pGRN145 as the template. (B) The amplified sequence of hTERT was cloned into EcoRI/NotI sites of pCDH lentivector plasmid. (C) Reverse transcription-PCR showed positive mRNA expression of hTERT and pCDH in HUVEC-TERTs. (D) Telomeric repeat amplification protocol assay suggested high telomerase activity in HUVEC-TERTs (Note: 293 cell extracts were the positive control provided by the manufacturer. $\left.{ }^{*} \mathrm{P}<0.05\right)$.

denatured and hybridized to microplate modules and detected by ELISA assay.

Tube formation assay. The tube formation assay was conducted to examine whether HUVEC-TERTs with extended passages could form vascular tubes. Namely, Matrigel basement membrane matrix (Becton-Dickinson and Company, Franklin Lakes, NJ, USA) was thawed at $4^{\circ} \mathrm{C}$ and coated at pre-cooled 6-well plates (17). Suspensions of $1 \times 10^{5}$ cells were plated on each well and incubated in $5 \% \mathrm{CO}_{2}$ at $37^{\circ} \mathrm{C}$ overnight. Tube formation was observed under a microscope.

Senescence-associated $\beta$-galactosidase staining. The staining was performed with senescence $\beta$-galactosidase staining kit 
(Beyotime Institute of Biotechnology, Shanghai, China) according to the protocol. Briefly, cells were washed and fixed, followed by the staining with working mixture and incubation at $37^{\circ} \mathrm{C}$ without $\mathrm{CO}_{2}$ overnight. Cells were observed with the camera-equipped microscope.

Cell proliferation studies. Long-term cell growth in vitro was observed by cell number count and population doubling level (PDL) measurement. Cumulative population doubling to each passage was calculated using the formula: PD (n) = $\log _{2}\left(N_{n} / N_{0}\right)$, (n, passage; $N_{n}$, cell number at passage $n ; N_{0}$, cell number at passage 0$)$.

Western blot analysis. VEGFR-II and integrin $\alpha 5$, the two identified proteins responsible for cross reaction in the antiangiogenic immunity, were detected by western blot analysis (5). Namely, proteins from HUVEC-TERTs and HUVECs (30 $\mu \mathrm{g} /$ lane) were fractionated on an $8 \%$ SDS-PAGE gel and electroblotted onto a polyvinylidene difluoride membrane (Bio-Rad Laboratories, Hercules, CA, USA). The membrane blots were blocked in 5\% non-fat dry milk, and incubated with anti-human VEGFR-II (1:500; Abcam, Cambridge, UK) or anti-human integrin $\alpha 5$ (1:500; R\&D Systems, Minneapolis, $\mathrm{MN}, \mathrm{USA}$ ) at $4^{\circ} \mathrm{C}$ overnight. Protein bands were then probed with HRP conjugated secondary antibody (Sigma-Aldrich) at $37^{\circ} \mathrm{C}$ for $1 \mathrm{~h}$ and were visualized on the chemiluninescence imager (Beijing Sage Creation Science Co., Ltd., Beijing, China).

Vaccine preparation and animal tumor challenge. To prepare the vaccine, HUVEC-TERTs (passage 30-35) and primary HUVECs (passage 3-5) were harvested and washed three times by M199 medium without fetal bovine or antibiotics. The cells were then irradiated by $100 \mathrm{~Gy}$ of X-ray, when no proliferation of cells was observed.

Six- to eight-week-old female C57BL/6 mice and BALB/C mice were used for lung cancer model (LL2) and colorectal cancer model (CT26), respectively. Mice were housed in a specific-pathogen-free facility and were randomly divided into 3 groups (10 for each group). In preventive immunization, each group was s.c. injected with $1 \times 10^{6}$ irradiated HUVEC-TERTs, HUVECs or NS alone (100 $\mu \mathrm{l} /$ dose) at day 0 , day 14 and day 21 in the left flank. At day 28, mice were s.c. injected with $5 \times 10^{5}$ tumor cells in the right flank. In therapeutic immunization, mice were firstly challenged with $5 \times 10^{5}$ tumor cells, and were then immunized with HUVEC-TERTs, HUVECs $\left(1 \times 10^{6}\right.$ cells, $100 \mu \mathrm{l} /$ dose) or NS alone once a week for consecutive 4 weeks $(18,19)$. The tumor volume was estimated every 3 days by the following formula: Tumor volume $=0.52 \mathrm{xaxb}^{2}$, where a represented the longer diameter and $b$ represented the shorter diameter (20). All animal experiments were approved by the Institutional Animal Care and Treatment Committee of Sichuan University in China.

ELISA. To evaluate the activation of $\mathrm{IgG}$ and other regulatory factors after immunization, ELISA assays were performed in mouse sera and spleen lymphocyte supernatants. As for HUVEC-neutralizing IgG titer, mouse sera were obtained from the orbital veins of mice 7 days after the third immunization of the preventive model. Mouse sera diluted serially by
PBS were then added to HUVEC-seeded 96-well plates $\left(1 \times 10^{4}\right.$ cells, $100 \mu \mathrm{l} /$ well) and labeled with horseradish peroxidase (HRP) anti-mouse IgG. Enzyme activity was visualized by TMB substrate and the absorbance was measured at $450 \mathrm{~nm}$ with an ELISA reader (Bio-Rad Laboratories).

Using sandwich precoated ELISA kits (Dakewe Biotech Co., Ltd., Beijing, China), TGF- $\beta$ and VEGF levels in the sera were tested 20 days after tumor inoculation of preventive model while IL-4 and INF- $\gamma$ levels in mouse lymphocyte supernatants were evaluated at day 7 after tumor inoculation of preventive model. For lymphocyte supernatant preparation, mice were sacrificed and spleen lymphocytes were extracted by lymphocyte separation medium (Dakewe Biotech) according to the manufacturer's recommendations. Cells were cultured in a 6-well plate $\left(1 \times 10^{7}\right.$ cells/well with $2 \mathrm{ml}$ serum-free Dulbecco's modified Eagle's medium) and supernatants were harvested after $48 \mathrm{~h}$ of culture. Mouse sera or lymphocyte supernatants were then added into the precoated microplates and incubated with biotin-labeled antibodies as well as streptavidin-labeled HRP. The reagents were visualized with TMB substrate for $30 \mathrm{~min}$ at $37^{\circ} \mathrm{C}$ without light and were measured at $450 \mathrm{~nm}$ with an ELISA reader.

Cytotoxic T-lymphocyte assay. Lymphocytes extracted from mouse spleens at day 7 after the third immunization were used as effector cells to incubate with HUVECs or LL2s in a 96-well plate for $4 \mathrm{~h}$ at $37^{\circ} \mathrm{C}$. Effector: target ratios were 12.5:1, 25:1, 50:1 and 100:1. Lactate dehydrogenase released by lysed cells in supernatants was measured by LDH cytotoxicity assay (GenMed Scientifics, DX Zoetermeer, The Netherlands). The cytotoxicity of lymphocytes were calculated with the following formula: \% cytotoxicity = (experimental O.D. - effector spontaneous O.D. - target spontaneous O.D.)/(maximal O.D. - target spontaneous O.D.) x $100 \%$.

Flow cytometric analysis. To test the activation of spleen cells, spleen lymphocytes at day 7 after the third immunization were labeled with anti-CD4-APC, anti-CD8-FITC and anti-CD69-PE or anti-CD4-APC, anti-CD8-FITC and antiIFN- $\gamma$-PE at $4^{\circ} \mathrm{C}$ for $30 \mathrm{~min}$. Stained cells were washed twice with $2 \mathrm{ml}$ PBS and resuspended in $500 \mu \mathrm{l}$ PBS. The percentages of activated cells were calculated on FACSCalibur using CellQuest software (Becton-Dickinson and Company).

To explore possible tumor microenvironment changes, mice were sacrificed 20 days after tumor inoculation of the preventive model. Tumor tissues were digested by $0.1 \%$ collagenase at $37^{\circ} \mathrm{C}$ for $3 \mathrm{~h}$. Tumor cells were then labeled with anti-CD4-APC, anti-CD8-FITC and anti-CD69-PE (activated $\mathrm{CD}^{+}{ }^{+}$or $\mathrm{CD}^{+} \mathrm{T}$ cells) or anti-CD25-PE and anti-Foxp3-FITC (regulatory $\mathrm{T}$ cells) or anti-CD11b-PE and anti-Gr-1-FITC (myeloid-derived suppressor cells) at $4^{\circ} \mathrm{C}$ for $30 \mathrm{~min}$ (21-23). Stained cells were analyzed as described above.

Immunohistochemistry. To explore whether HUVEC-TERTs could inhibit angiogenesis in vivo, paraffin sections of tumor tissues were incubated overnight with the vascular endothelial antibody against CD34 (1:500; Abcam), followed by incubation with biotinylated secondary antibody at $37^{\circ} \mathrm{C}$ for $40 \mathrm{~min}$ and streptavidin-biotin complex at $37^{\circ} \mathrm{C}$ for $40 \mathrm{~min}$ (ZSGB-Bio, Beijing, China). The numbers of CD34-positive microvessels 

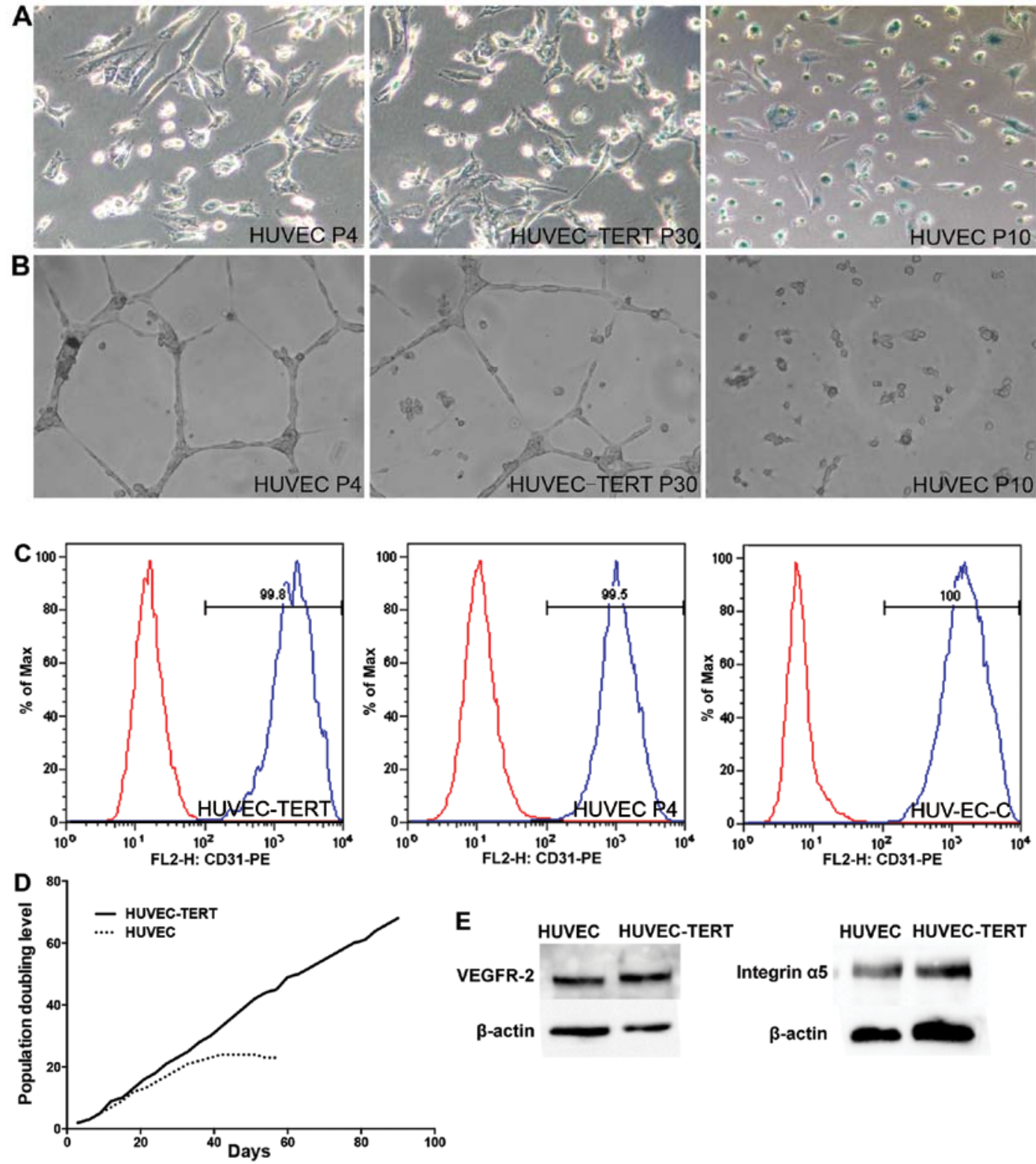

Figure 2. Endothelial characteristics of HUVEC-TERTs. (A) Senescence-associated $\beta$-galactosidase staining. Passage-30 HUVEC-TERTs and passage-4 HUVECs presented no obvious staining while passage-10 HUVECs showed massive staining (blue cells). (B) Tube forming on Matrigel. Passage-30 HUVECTERTs and passage-4 HUVECs were able to form vascular tubes while passage-10 HUVECs lost the endothelial tube formation ability. (C) Flow cytometric analysis. The expression of specific endothelial marker CD31 was tested in HUVEC-TERTs, HUVECs and HUV-EC-Cs (Note: red curves are the anti-IgG-PE isotopes set as negative controls). (D) Growth curves of HUVEC-TERTs and HUVECs. HUVECs entered senescence after 24 PDs while HUVEC-TERTs were still proliferative after 70 PDs. (E) Western blot analysis. Passage-30 HUVEC-TERTs resembled young primary HUVECs in the expression of VEGFR-II and integrin $\alpha 5$, the two identified proteins responsible for cross reaction in the anti-angiogenic immunity.

and infiltrating lymphocytes were microscopically examined in the high-power field of view at a magnification of x 200 .

Statistical analysis. Statistical comparisons were made by 2-tailed Student's t-test or log-rank test. Differences were considered statistically significant at $\mathrm{P}<0.05$.

\section{Results}

hTERT-transfected HUVECs show an extended life span and maintain endothelial characteristics. The ectopic expression of hTERT in HUVECs was tested at mRNA and protein levels. RT-PCR showed that both hTERT mRNA and vector pCDH mRNA were expressed after transfection (Fig. 1C). In TRAP-
ELISAs, while primary HUVECs presented no obvious telomerase activity, the telomerase level of HUVEC-TERTs was significantly higher $(\mathrm{P}<0.05)$ and was $96 \%$ of that of the 293 cells, the embryonic kidney tumor cell line (Fig. 1D). These data suggested that HUVECs were successfully transduced with human telomerase cDNA and HUVEC-TERTs presented high telomerase activity.

In contrast to primary HUVECs, which showed marked $\beta$-galactosidase production and loss of endothelial tube formation ability at passage 10, passage-30 HUVEC-TERTs presented no obvious $\beta$-galactosidase staining and were able to form vascular tubes on Matrigel (Fig. 2A and B). In addition, HUVECs went into growth plateau after 24 PDs while HUVEC-TERTs were still proliferative after 70 PDs (Fig. 2D). 

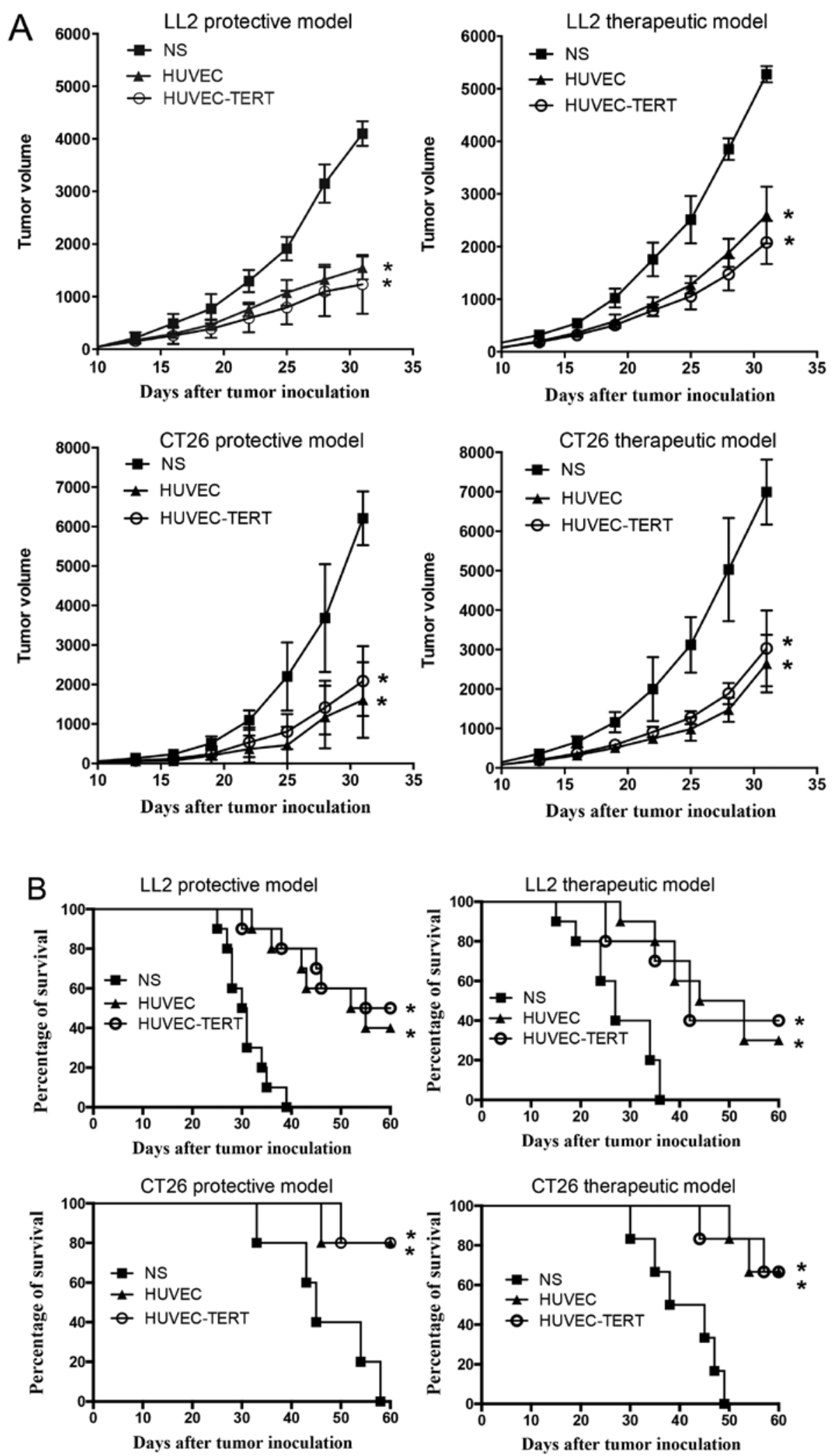

Figure 3. Tumor volumes and mouse survival. (A) Tumors in HUVEC-TERTs and HUVECs immunized mice grew significantly slower than those of the NS groups in LL2 model and CT26 model both protectively and therapeutically. (B) Tumor-bearing mice survived significantly longer in HUVEC-TERTs and HUVECs treated mice compared with NS groups ( $\left.{ }^{*} \mathrm{P}<0.05\right)$.

Moreover, HUVEC-TERTs expressed CD31, a specific endothelial marker that was evaluated by flow cytometric analysis (Fig. 2C) (24). Passage-30 HUVEC-TERTs also resembled young primary HUVECs in the expression of VEGFR-II and integrin $\alpha 5$, the two identified proteins responsible for cross reaction in the anti-angiogenic immunity (Fig. 2E). Thus, hTERT transfection prolonged the life span of HUVECs while remained their endothelial characteristics. 

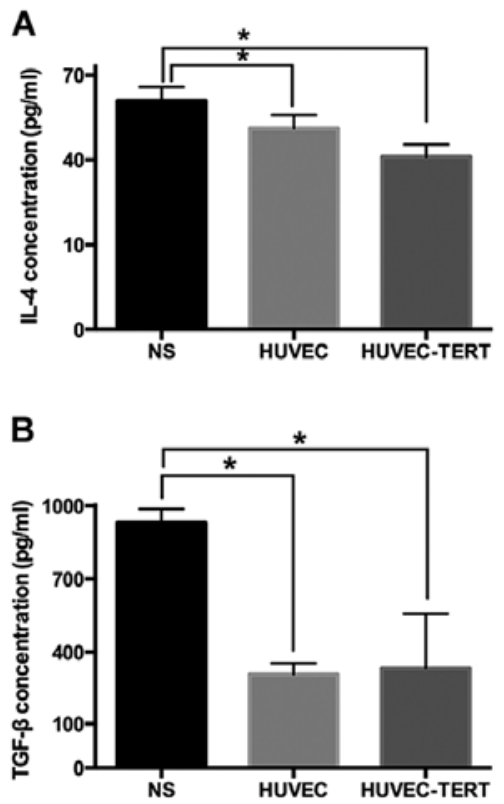

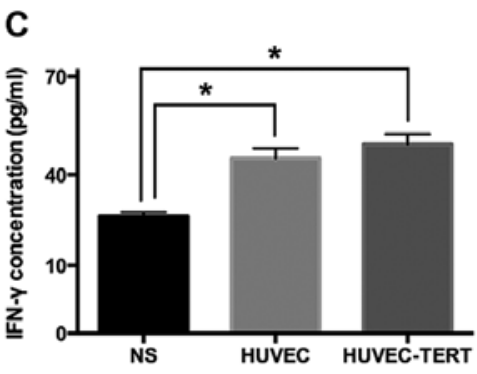

D

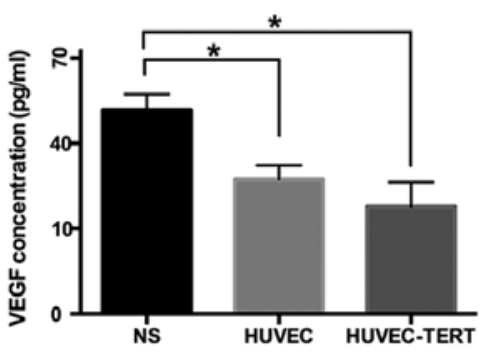

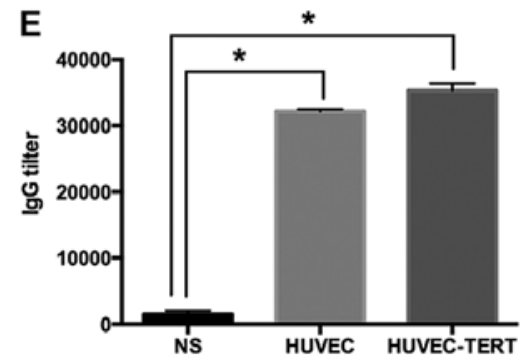
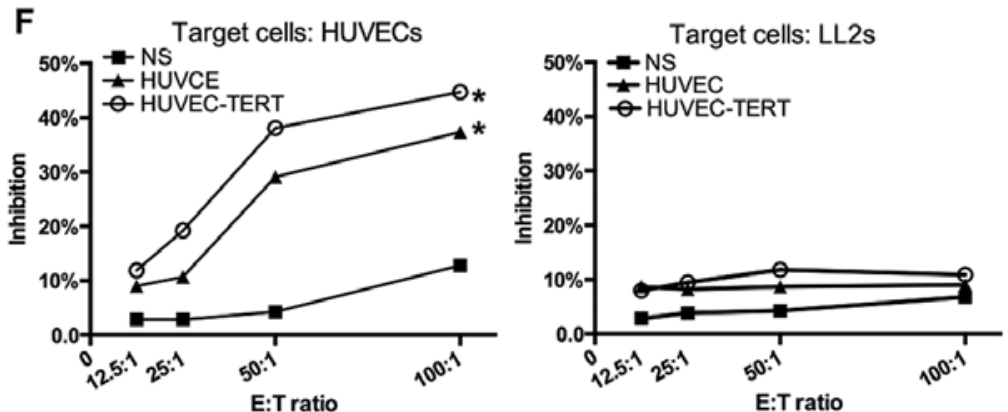

Figure 4. ELISA assays of immune mediators and IgG titer and CTL assay. (A and C) ELISA of lymphocyte supernatants 7 days after tumor inoculation. IL-4 was decreased while INF- $\gamma$ was increased in HUVEC-TERTs treated mice. (B and D) ELISA of mouse sera 20 days after tumor inoculation. TGF- $\beta$ and VEGF concentrations were decreased in HUVEC-TERT group. (E) Production of neutralizing IgG against HUVECs was higher in HUVEC-TERTs and HUVECs treated mouse sera. (F) CTL assay based on LDH release. Spleen lymphocytes from both HUVEC-TERTs and HUVECs treated mice were cytotoxic specifically and dose-dependently to HUVECs ( $\mathrm{P}<0.05)$.

HUVEC-TERTs induced protective and therapeutic antitumor immunity. To confirm whether hTERT-immortalized HUVECs retained antitumor immunity similarly to primary HUVECs, both protective and therapeutic mouse models were established in HUVEC-TERT, HUVEC and NS groups. As shown in Fig. 3A, tumors in HUVEC-TERTs and HUVECimmunized mice grew significantly slower compared with NS group. Thirty-one days after tumor inoculation, tumor inhibition rates in HUVEC-TERT group and HUVEC group were 69 . and $62.3 \%$ in the protective LL2 model and were 66.0 and $74.0 \%$ in the protective CT26 model. In therapeutic models, treatment with HUVEC-TERTs and HUVECs also significantly repressed tumor growth. In addition, survival rates of
HUVEC-TERTs and HUVECs treated mice were significantly higher than controls in both tumor models (Fig. 3B).

The production of immunoglobulin and regulatory factors in mouse sera. The IgG titers tested by ELISAs in HUVETTERT group and HUVEC group were 1:35,600 and 1:32,200, respectively (Fig. 4E). Moreover, compared with NS group, lymphatic secretion of IFN- $\gamma$ (secreted mainly by Th1) was increased while IL-4 (secreted mainly by Th2) was decreased significantly in both groups $(\mathrm{P}<0.05) 7$ days after tumor inoculation $(25,26)$. Whereas, VEGF and TGF- $\beta$, the two important immunosuppressive mediators, were diminished $(\mathrm{P}<0.05)$ in sera 20 days after tumor inoculation (Fig. 4A and D) $(27,28)$. 

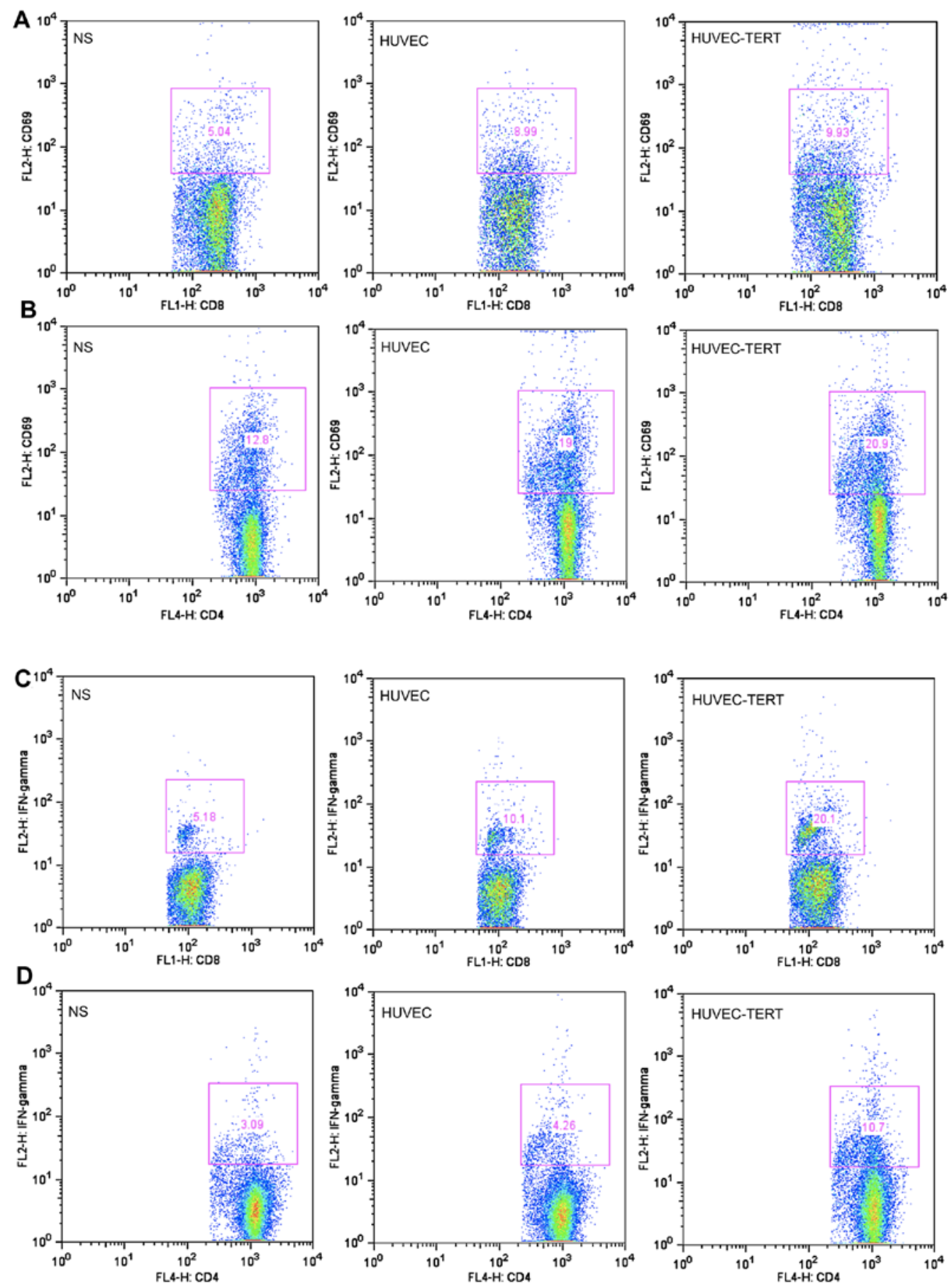

Figure 5. Flow cytometric analysis of spleen lymphocytes and tumor tissues. (A-D) Flow cytometric analysis of spleen lymphocytes 7 days after third immunization. (A and B) Both activated CD8 ${ }^{+} \mathrm{T}$ cells $\left(\mathrm{CD} 8^{+} \mathrm{CD} 69^{+}\right)$and $\mathrm{CD} 4^{+} \mathrm{T}$ cells $\left(\mathrm{CD} 4^{+} \mathrm{CD} 69^{+}\right)$were elevated in HUVEC-TERT group. (C and D) Percentages of IFN- $\gamma$ expressing $C D 8^{+} \mathrm{T}$ cells and $\mathrm{CD} 4^{+} \mathrm{T}$ cells of spleen lymphocytes were significantly larger in HUVEC-TERT group compared with NS group.

These data suggested that immunization with irradiated HUVEC-TERTs increased the production of immunoglobulin against endothelial cells and changed secretion levels of immune mediators.

T lymphocytes from HUVEC-TERT-immunized mice are cytotoxic to HUVECs, but not to tumor cells. In CTL assay based on LDH release of lysed target cells, T lymphocytes isolated from spleens of HUVEC-TERTs and HUVECs vaccinated mice were found to kill HUVECs in vitro dose-dependently. At 100:1 E:T ratio, the cytotoxicity of HUVEC-TERT group was 3.5 times that of the NS group. However, no obvious cytotoxicity to LL2 tumor cells was observed in any of the three groups (Fig. 4F), suggesting that lymphocytes were specifically and dose-dependently cytotoxic to the endothelium in the antitumor immunity.

Spleen lymphocytes were activated by HUVEC-TERT immunization. The flow cytometric analysis showed that HUVEC-TERT group and HUVEC group presented higher ratios of activated $\mathrm{CD} 4^{+} \mathrm{T}$ cells $\left(\mathrm{CD} 4^{+} \mathrm{CD} 69^{+}\right)$and activated $\mathrm{CD} 8^{+} \mathrm{T}$ cells $\left(\mathrm{CD} 8^{+} \mathrm{CD} 69^{+}\right)$at day 7 after the third immuniza- 
E
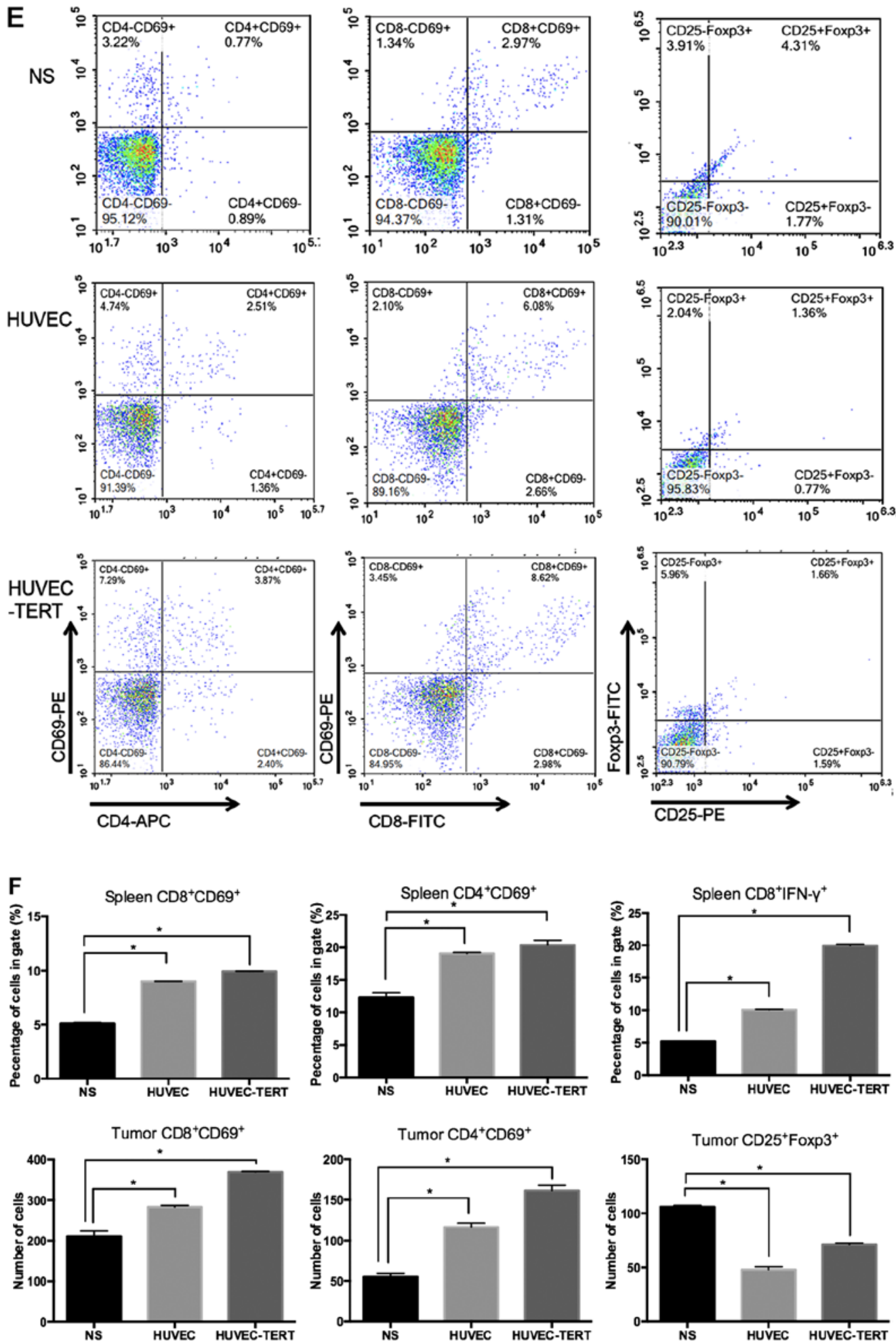
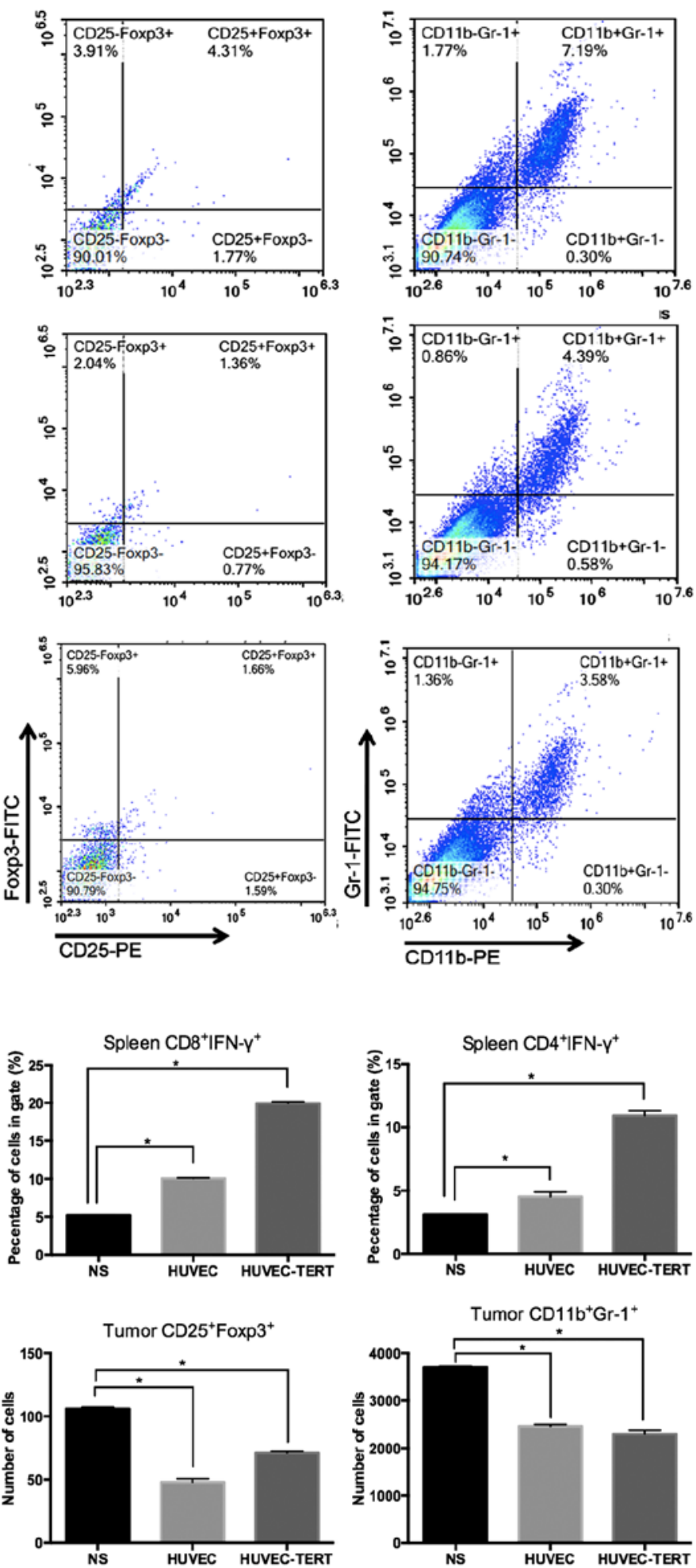

Figure 5. Continued. (E) Flow cytometric analysis of digested tumor tissues 20 days after tumor inoculation of the preventive model. Larger numbers of activated $\mathrm{CD}^{+} \mathrm{T}$ cells $\left(\mathrm{CD} 8^{+} \mathrm{CD} 9^{+}\right)$and $\mathrm{CD} 4^{+} \mathrm{T}$ cells $\left(\mathrm{CD} 4^{+} \mathrm{CD} 69^{+}\right)$were infiltrated while Tregs $\left(\mathrm{CD} 25^{+} \mathrm{Foxp} 3^{+}\right)$and $\mathrm{MDSCs}\left(\mathrm{CD} 11 \mathrm{~b}^{+} \mathrm{Gr}-1^{+}\right)$were downregulated in tumor tissues of HUVEC-TERT group. (F) Statistical analysis of flow cytometry (Note: positive cells in tumor tissues were counted in numbers per 10,000-cell storage because their percentages were small. $\left.{ }^{*} \mathrm{P}<0.05\right)$.

tion (Fig. 5A, B and F). In accordance with ELISAs, the flow cytometric analysis also revealed a significant increase in the secretion of IFN- $\gamma$ in $\mathrm{CD}^{+} \mathrm{T}$ cells and $\mathrm{CD}^{+} \mathrm{T}$ cells (Fig. 5C, $\mathrm{D}$ and $\mathrm{F}$ ). The above results suggested that subsets of both $\mathrm{CD} 4^{+} \mathrm{T}$ cells and $\mathrm{CD} 8^{+} \mathrm{T}$ cells were activated in the HUVECTERT-induced antitumor immunity.
HUVEC-TERTs increase lymphocyte infiltration and impair regulatory $T$ cells (Tregs) and myeloid-derived suppressor cell (MDSCs) infiltration in tumor tissues. To test possible tumor microenvironmental changes despite anti-angiogenesis, flow cytometric analysis was conducted on digested tumor tissues. As to tumor infiltrating lymphocytes, the numbers of 

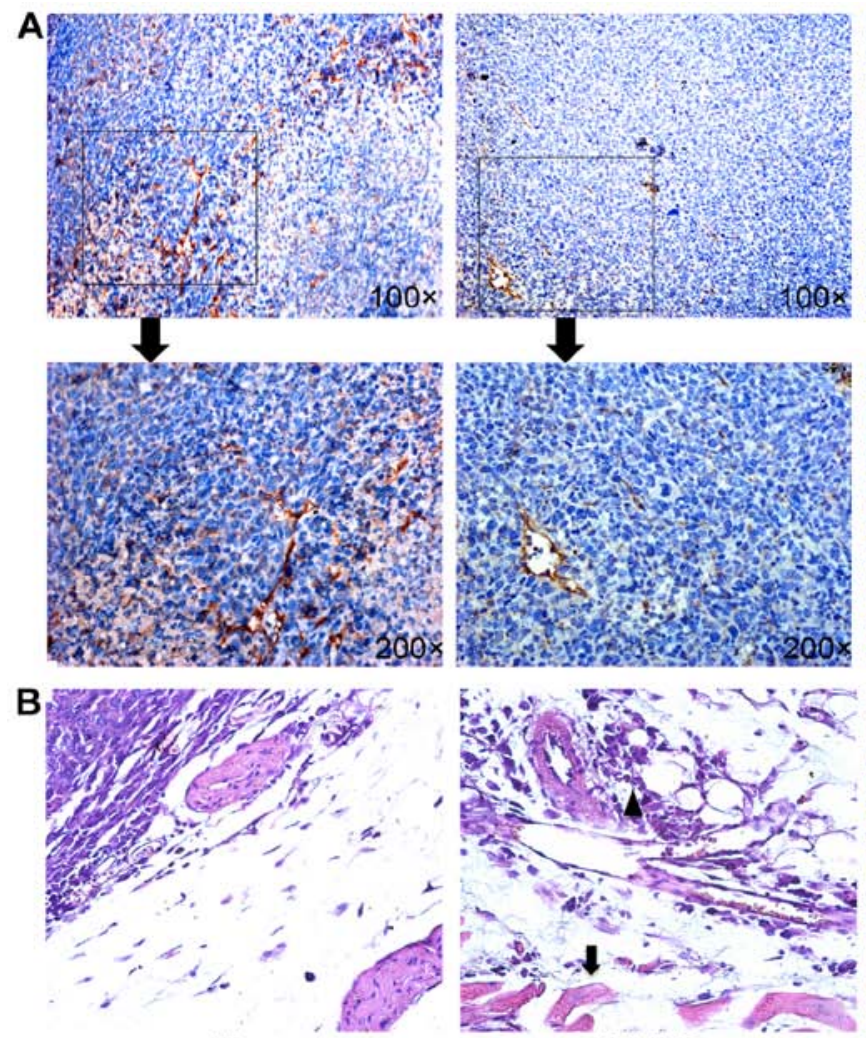

NS

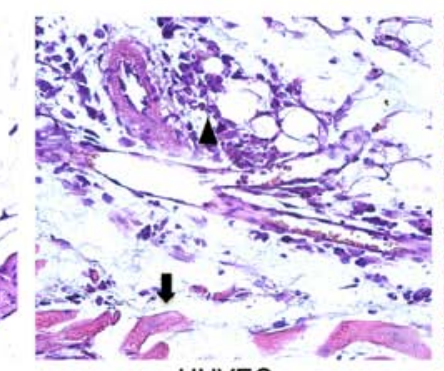

HUVEC
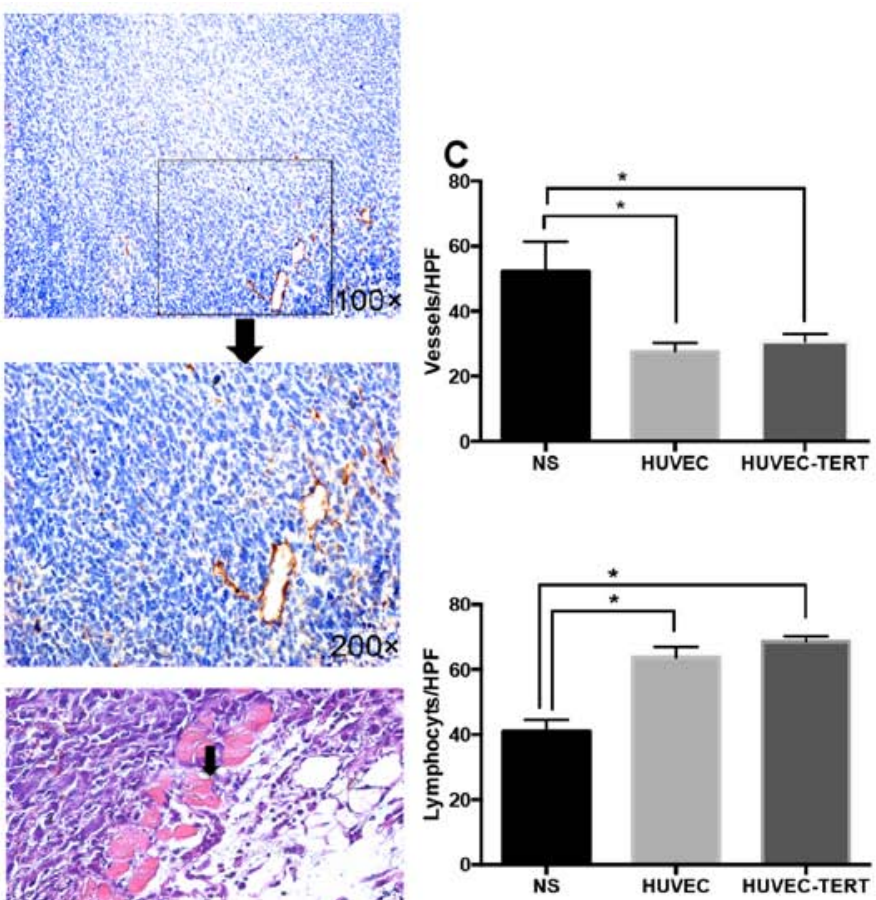

Figure 6. Immunohistochemistry and H\&E staining of tumors. (A) IHC of tumor microvessels labeled with endothelial anti-CD34. Microvessels were significantly diminished in HUVEC-TERT and HUVEC groups (observed at x100 and x200 magnification). (B) H\&E staining presented significant symptoms of hemorrhage (arrow) and inflammatory infiltrates (triangle) in HUVEC-TERT and HUVEC groups while tumor cells of NS group were highly proliferative, with no obvious symptoms of hemorrhage or inflammatory infiltrates. (C) Mean numbers of microvessels and infiltrating lymphocytes at high power field of $\mathrm{x} 200\left({ }^{*} \mathrm{P}<0.05\right)$.

activated $\mathrm{CD} 4^{+} \mathrm{T}$ cells $\left(\mathrm{CD} 4^{+} \mathrm{CD} 69^{+}\right)$and activated $\mathrm{CD} 8^{+} \mathrm{T}$ cells $\left(\mathrm{CD} 8^{+} \mathrm{CD}^{+} 9^{+}\right)$per 10,000 -cell storage were significantly larger in HUVEC-TERT group and HUVEC group compared with NS group. Besides, regulatory T cells $\left(\mathrm{CD} 25^{+} \mathrm{Foxp} 3^{+}\right)$, an important type of immunosuppressive cells, were significantly decreased in both groups (29). Furthermore, myeloid-derived suppressor cells (MDSCs), which were related to antigenspecific $\mathrm{CD}^{+} \mathrm{T}$ cell tolerance, were also significantly suppressed in tumor tissues of HUVEC-TERTs and HUVECs treated mice (Fig. 5E and F) (30). These data suggested that in addition to vascular damage, tumor microenvironment was also regulated in the HUVEC-TERT-induced antitumor immunity.

Immunization with HUVEC-TERTs inhibits angiogenesis but shows no damage to major organs. Paraffin sections of tumor tissues showed that HUVEC-TERT and HUVEC groups experienced a significant decrease in $\mathrm{CD} 34^{+}$microvessels (Fig. 6A and C) (31). H\&E staining of tumor sections presented symptoms of stromal hemorrhage and inflammatory infiltrates in HUVEC-TERT and HUVEC groups while tumor cells of NS group were highly proliferative, with no obvious symptoms of hemorrhage or inflammatory infiltrates (Fig. 6B and C). Notaby, the results of H\&E staining also showed that mice vaccinated with HUVEC-TERTs and HUVECs had no pathologic changes in heart, liver, spleen, lungs and kidneys (data not shown). These data indicated that HUVEC-TERTs inhibited tumor angiogenesis and had no toxicity to major organs.

\section{Discussion}

HUVECs have been proved to be effective in the anti-angiogenic immunity against cancer but their restricted ability to proliferate and expensive culture condition limit their application on a large scale (32). In the present study, we immortalized primary HUVECs by virally introducing hTERT genes and explored whether the antitumor immunity could be maintained through vaccination with hTERT-expressing HUVECs. The present study found that hTERT-expressing HUVECs displayed high telomerase activity at both transcription and protein levels. Passage-30 HUVEC-TERTs presented no obvious $\beta$-galactosidase staining and were able to form vascular tubes on matrigel. They also expressed endothelial specific marker CD31 and the two identified proteins for cross reaction (VEGFR-II and integrin $\alpha 5$ ). This proved that virally introducing hTERT cDNA was an effective method to elongate the life span of primary HUVECs while maintaining their endothelial characteristics.

HUVEC-TERTs were found to elicit both protective and therapeutic antitumor immunity in tumor-bearing mice and we believed that the previously reported anti-angiogenesis was still responsible due to the following reasons: firstly, immunohistochemistry revealed significantly less CD34 posi- 
tive microvessels in HUVEC-TERT group; secondly, ELISAs tested higher production of neutralizing IgG against HUVECs from HUVEC-TERTs treated mouse sera; thirdly, CTL assay showed that spleen lymphocytes extracted from HUVECTERT-treated mice were dose-dependently and specifically cytotoxic to HUVECs; fourthly, immune suppressive mediators that mainly prompt angiogenesis were downregulated. TGF- $\beta$, which was secreted by endothelial cells, tumor cells and tumor-associated macrophages was decreased while VEGF, which was mainly produced by endothelial cells, was also diminished significantly (27).

The subsets of activated T lymphocytes in the antitumor immunity were further elucidated. The flow cytometric analysis of spleens demonstrated that both $\mathrm{CD} 4^{+}$and $\mathrm{CD} 8^{+} \mathrm{T}$ lymphocytes were almost doubled in HUVEC-TERT group compared with NS group. This was different from the previous report that mice were not protected from tumor challenge only when they were depleted of $\mathrm{CD} 4^{+} \mathrm{T}$ lymphocytes and vaccinated with xenogeneic endothelial cells (5). We suspected that this contradiction was caused by the different vaccinepreparing methods. In previous studies, methods to prepare HUVEC vaccines differed from $3 \%$ paraformaldehyde fixation, $0.025 \%$ glutaraldehyde fixation to no special treatment by using viable HUVECs $(5,26,32)$. In the present study, we treated HUVEC-TERTs and HUVECs with X-ray, when no proliferation was observed. As is known to us, irradiation was able to restrict cell proliferation, increase antigen presentation and enhance cytotoxic T lymphocyte recognition (18). This might be the reason that the present study revealing a stronger cellular immunity.

Despite $\mathrm{CD}^{+}{ }^{+}$and $\mathrm{CD} 8^{+} \mathrm{T}$ lymphocytes, Th1 and Th2 also played a role since the Th1 cytokine IFN- $\gamma$ was increased while the Th2 cytokine IL-4 was decreased, which was consistent with the previous observation that IFN- $\gamma$ production was reduced and IL-4 production was enhanced in a variety of human malignancies, including melanoma, gastric cancer, lung cancer, glioblastoma, nasopharyngeal carcinoma, colorectal cancer and head and neck cancer $(33,34)$.

In recent years, the tumor microenvironment is being increasingly recognized as a key factor in multiple stages of tumor progression. Inappropriate activation of the stroma, which involves migration of stromal cells, remodeling of matrix, and expansion of vasculature, is able to convert the physiological microenvironment into a pathological entity (35). For instance, it was proven that tumors employed various strategies to recruit MDSCs, a major component of tumor-adjacent stroma, to positively regulate tumor development (36). It was also reported that MDSCs could stimulate Tregs, inhibit $\mathrm{T}$ lymphocytes proliferation and promote angiogenesis by secreting VEGF, TGF- $\beta$ and FGF (37). In the present study, the flow cytometric analysis of tumor tissues revealed that both MDSCs and Tregs were decreased while the $\mathrm{H} \& \mathrm{E}$ staining showed intense $\mathrm{CD}^{+}$and $\mathrm{CD} 8^{+} \mathrm{T}$ cells migration in the stroma of HUVEC-TERT group, indicating that the antitumor immunity of HUVEC-TERTs involve both anti-angiogenesis and the regulation of other factors in the tumor microenvironment.

To the best of our knowledge, the present study is the first to confirm the antitumor immunity of irradiated hTERTimmortalized HUVECs. Both anti-angiogenesis and tumor microenvironmental regulation were responsible for the antitumor activity. Transducing hTERT genes might be a new strategy to allow HUVECs to be applied on a large scale in tumor immunotherapy.

\section{Acknowledgements}

The present study was supported by the National Natural Science Foundation of China (nos. 81372246 and 81123003) and the National High Technology Research and Development Program of China (no. 2014AA020708).

\section{References}

1. Okaji Y, Tsuno NH, Saito S, Yoneyama S, Tanaka M, Nagawa H and Takahashi K: Vaccines targeting tumour angiogenesis - a novel strategy for cancer immunotherapy. Eur J Surg Oncol 32: 363-370, 2006.

2. Boehm T,Folkman J, Browder T and O'Reilly MS: Antiangiogenic therapy of experimental cancer does not induce acquired drug resistance. Nature 390: 404-407, 1997.

3. Dranoff G, Jaffee E, Lazenby A, Golumbek P, Levitsky H, Brose K, Jackson V, Hamada H, Pardoll D and Mulligan RC: Vaccination with irradiated tumor cells engineered to secrete murine granulocyte-macrophage colony-stimulating factor stimulates potent, specific, and long-lasting anti-tumor immunity. Proc Natl Acad Sci USA 90: 3539-3543, 1993.

4. Liu JY, Wei YQ, Yang L, Zhao X, Tian L, Hou JM, Niu T, Liu F, Jiang Y, Hu B, et al: Immunotherapy of tumors with vaccine based on quail homologous vascular endothelial growth factor receptor-2. Blood 102: 1815-1823, 2003.

5. Wei YQ, Wang QR, Zhao X, Yang L, Tian L, Lu Y, Kang B, Lu CJ, Huang MJ, Lou YY, et al: Immunotherapy of tumors with xenogeneic endothelial cells as a vaccine. Nat Med 6: 1160-1166, 2000.

6. Chen XY, Zhang W, Zhang W, Wu S, Bi F, Su YJ, Tan XY, Liu JN and Zhang J: Vaccination with viable human umbilical vein endothelial cells prevents metastatic tumors by attack on tumor vasculature with both cellular and humoral immunity. Clin Cancer Res 12: 5834-5840, 2006.

7. Okaji Y, Tsuno NH, Tanaka M, Yoneyama S, Matsuhashi M, Kitayama J, Saito S, Nagura Y, Tsuchiya T, Yamada J, et al: Pilot study of anti-angiogenic vaccine using fixed whole endothelium in patients with progressive malignancy after failure of conventional therapy. Eur J Cancer 44: 383-390, 2008.

8. Chang MW, Grillari J, Mayrhofer C, Fortschegger K, Allmaier G, Marzban G, Katinger H and Voglauer R: Comparison of early passage, senescent and hTERT immortalized endothelial cells. Exp Cell Res 309: 121-136, 2005.

9. Böcker W, Yin Z, Drosse I, Haasters F, Rossmann O, Wierer M, Popov C, Locher M, Mutschler W, Docheva D, et al: Introducing a single-cell-derived human mesenchymal stem cell line expressing hTERT after lentiviral gene transfer. J Cell Mol Med 12: 1347-1359, 2008

10. Freedman DA and Folkman J: Maintenance of G1 checkpoint controls in telomerase-immortalized endothelial cells. Cell Cycle 3: 811-816, 2004.

11. Bodnar AG, Ouellette M, Frolkis M, Holt SE, Chiu CP, Morin GB, Harley CB, Shay JW, Lichtsteiner S and Wright WE: Extension of life-span by introduction of telomerase into normal human cells. Science 279: 349-352, 1998.

12. Zhu J, Wang H, Bishop JM and Blackburn EH: Telomerase extends the lifespan of virus-transformed human cells without net telomere lengthening. Proc Natl Acad Sci USA 96: 3723-3728, 1999.

13. Anno K, Hayashi A, Takahashi T, Mitsui Y, Ide T and Tahara H: Telomerase activation induces elongation of the telomeric singlestranded overhang, but does not prevent chromosome aberrations in human vascular endothelial cells. Biochem Biophys Res Commun 353: 926-932, 2007.

14. Young AT, Lakey JR, Murray AG, Mullen JC and Moore RB: In vitro senescence occurring in normal human endothelial cells can be rescued by ectopic telomerase activity. Transplant Proc 35: 2483-2485, 2003.

15. von Zglinicki T: Oxidative stress shortens telomeres. Trends Biochem Sci 27: 339-344, 2002. 
16. Yang J, Chang E, Cherry AM, Bangs CD, Oei Y, Bodnar A Bronstein A, Chiu CP and Herron GS: Human endothelial cell life extension by telomerase expression. J Biol Chem 274 26141-26148, 1999.

17. Yang J, Nagavarapu U, Relloma K, Sjaastad MD, Moss WC, Passaniti A and Herron GS: Telomerized human microvasculature is functional in vivo. Nat Biotechnol 19: 219-224, 2001.

18. Li Y, Shen G, Nie W, Li Z, Sang Y, Zhang B and Wei Y: Irradiated tumor cells of lipopolysaccharide stimulation elicit an enhanced anti-tumor immunity. J Cancer Res Clin Oncol 140: 1815-1823, 2014.

19. Zhao Z, Yao Y, Ding Z, Chen X, Xie K, Luo Y, Zhang J, Chen X, Wu X, Xu J, et al: Antitumour immunity mediated by mannanmodified adenovirus vectors expressing VE-cadherin. Vaccine 29: 4218-4224, 2011.

20. Zhou X, Li J, Wang Z, Chen Z, Qiu J, Zhang Y, Wang W, Ma Y, Huang N, Cui K, et al: Cellular immunotherapy for carcinoma using genetically modified EGFR-specific T lymphocytes. Neoplasia 15: 544-553, 2013

21. Gupta S, Marcel N, Sarin A and Shivashankar GV: Role of actin dependent nuclear deformation in regulating early gene expression. PLoS One 7: e53031, 2012.

22. Zhang C, Shan J, Lu J, Huang Y, Feng L, Long D, Li S, Li Q and Li Y: Combination of rapamycin and IL-2 do not affect antigen presentation ability of rat B cell and could promote Tregs proliferation and inhibitory activity. Cell Immunol 264: $180-185,2010$

23. Skabytska Y, Wölbing F, Günther C, Köberle M, Kaesler S, Chen KM, Guenova E, Demircioglu D, Kempf WE, Volz T, et al: Cutaneous innate immune sensing of Toll-like receptor 2-6 ligands suppresses $\mathrm{T}$ cell immunity by inducing myeloid-derived suppressor cells. Immunity 41: 762-775, 2014.

24. Li Y, Zhao YJ, Zou QY, Zhang K, Wu YM, Zhou C, Wang K and Zheng J: Preeclampsia does not alter vascular growth and expression of CD31 and vascular endothelial cadherin in human placentas. J Histochem Cytochem 63: 22-31, 2015.

25. Bürgler S, Gimeno A, Parente-Ribes A, Wang D, Os A, Devereux S, Jebsen P, Bogen B, Tjønnfjord GE and Munthe LA: Chronic lymphocytic leukemia cells express CD38 in response to Th1 cell-derived IFN- $\gamma$ by a T-bet-dependent mechanism. J Immunol 194: 827-835, 2015

26. Lohoff M, Giaisi M, Köhler R, Casper B, Krammer PH and Li-Weber M: Early growth response protein-1 (Egr-1) is preferentially expressed in T helper type 2 (Th2) cells and is involved in acute transcription of the Th2 cytokine interleukin-4. J Biol Chem 285: 1643-1652, 2010 .
27. Mulligan JK and Young MR: Tumors induce the formation of suppressor endothelial cells in vivo. Cancer Immunol Immunother 59: 267-277, 2010.

28. Blank S, Deck C, Dreikhausen L, Weichert W, Giese N, Falk C, Schmidt T and Ott K: Angiogenic and growth factors in gastric cancer. J Surg Res 194: 420-429, 2015.

29. Shatry A, Chirinos J, Gorin MA, Jones M and Levy RB: Targeting Treg cells in situ: Emerging expansion strategies for $\left(\mathrm{CD} 4{ }^{+} \mathrm{CD} 25^{+}\right)$regulatory T cells. Biol Blood Marrow Transplant 15: 1239-1243, 2009

30. Fernández A, Oliver L, Alvarez R, Fernández LE, Lee KP and Mesa C: Adjuvants and myeloid-derived suppressor cells: Enemies or allies in therapeutic cancer vaccination. Hum Vaccin Immunother 10: 3251-3260, 2014.

31. Xing X, Gu X, Ma T and Ye H: Biglycan up-regulated vascular endothelial growth factor (VEGF) expression and promoted angiogenesis in colon cancer. Tumour Biol 36: 1773-1780, 2015.

32. Okaji Y, Tsuno NH, Kitayama J, Saito S, Takahashi T, Kawai K, Yazawa K, Asakage M, Hori N, Watanabe T, et al: Vaccination with autologous endothelium inhibits angiogenesis and metastasis of colon cancer through autoimmunity. Cancer Sci 95: 85-90, 2004

33. Toomer KH and Chen Z: Autoimmunity as a double agent in tumor killing and cancer promotion. Front Immunol 5: 116, 2014.

34. Vierboom MP, Nijman HW, Offringa R, van der Voort EI, van Hall T, van den Broek L, Fleuren GJ, Kenemans P, Kast WM and Melief CJ: Tumor eradication by wild-type p53-specific cytotoxic T lymphocytes. J Exp Med 186: 695-704, 1997.

35. Chen F, Zhuang X, Lin L, Yu P, Wang Y, Shi Y, Hu G and Sun Y: New horizons in tumor microenvironment biology: Challenges and opportunities. BMC Med 13: 45, 2015.

36. Koh BI and Kang Y: The pro-metastatic role of bone marrowderived cells: A focus on MSCs and regulatory T cells. EMBO Rep 13: 412-422, 2012.

37. Pacini S: Deterministic and stochastic approaches in the clinical application of mesenchymal stromal cells (MSCs). Front Cell Dev Biol 2: 50, 2014. 